\title{
Online Nonlinear Identification of the Effect of Drugs in Anaesthesia Using a Minimal Parameterization and BIS Measurements
}

\author{
Margarida Martins da Silva, Student Member, IEEE, \\ Teresa Mendonça, Member, IEEE, and Torbjörn Wigren, Senior Member, IEEE
}

\begin{abstract}
This paper addresses the problem of modeling and identification of the Depth of Anaesthesia (DoA). It presents a new MISO Wiener model for the pharmacokinetics and pharmacodynamics of propofol and remifentanil, when jointly administered to patients undergoing surgery. The models most commonly used to describe the effect of drugs in the human body are overparameterized Wiener models. In particular, in an anaesthesia environment, the high number of patient-dependent parameters coupled with the insufficient excitatory pattern of the input signals (drug dose profiles) and the presence of noise make robust identification strategies difficult to find. In fact, in such clinical application the user cannot freely choose the input signals to enable accurate parameter identification. A new MISO Wiener model with only four parameters is hence proposed to model the effect of the joint administration of the hypnotic propofol and the analgesic remifentanil. An Extended Kalman Filter (EKF) algorithm was used to perform the nonlinear online identification of the system parameters. The results show that both the new model and the identification strategy outperform the currently used tools to infer individual patient response. The proposed DoA identification scheme was evaluated in a real patient database, where the DoA is quantified by the Bispectral Index Scale (BIS) measurements. The results obtained so far indicate that the developed approach will be a powerful tool for modeling and identification of anaesthetic drug dynamics during surgical procedures.
\end{abstract}

\section{INTRODUCTION}

The starting point for the mathematical modeling of physiological systems is usually the background knowledge of the chemical or physical laws present in such systems. In this sense, the deduced model variables are meaningful and can easily be interpreted as masses, volumes or even temperatures. The human response to the administration of drugs in anaesthesia is a good example of this. In what concerns depth of anaesthesia (DoA), the response for the administration of hypnotics and analgesics is commonly modeled as an high order pharmacokinetic/pharmacodynamic (PK/PD) Wiener model [1]. Here the parameters to be identified are compartment volumes, micro-constant rates for mass transfers between compartments and drug clearances in the human body.

System identification theory states that the identified model is a compromise between the richness of the data and the identifiability of the parameters [2]. The application of system identification strategies to identify each patient response to drug administration in anaesthesia is a particularly complex issue. This is because a common feature of such systems is the poor excitation, meaning that the input signals (administered drug doses) are not rich enough in frequency and amplitude to excite all modes of the system [3], as it is usually modeled. This is so since the data is obtained from the surgery room, where patient safety is the prime concern. The tradeoff between complexity and identifiability is therefore addressed with minimal modeling in the present paper.

The effect of drugs in the human body can often be modeled by a linear mixing dynamics, in cascade with a static nonlinear function that describes the effect of the drug. As a consequence of this Wiener

M. M. Silva and T. Mendonça are with the Departamento de Matemática, Faculdade de Ciências da Universidade do Porto, Rua do Campo Alegre, 4169-007 Porto, Portugal, and with the UI\&D Matemática e Aplicações, Departamento de Matemática, Campus Universitário de Santiago, 3810-193 Aveiro, Portugal (e-mail: margarida.silva,tmendo@fc.up.pt)

T. Wigren is with the Division of Systems and Control, Department of Information Technology, Uppsala University, Box 337, SE-751 05 Uppsala, Sweden (e-mail: torbjorn.wigren@it.uu.se) 
structure (a linear block in series with a static nonlinearity [4]), the prediction of human response to drugs cannot be accurately performed by linear models [5], [6]. Moreover, and in order to address the problem of having to deal with a model with many parameters, [7] presents a simplification of the model structure. In [7] it is also pointed out that to obtain a good modeling of the response, a good estimation of the PK model is needed. This fact gives rise to the problem of selecting the best structure to model the real cases. This idea was further developed in [8], where excellent results were obtained by nonlinear recursive identification of the neuromuscular blockade level using a two parameter model.

The contribution of this paper is to present a new method for nonlinear identification of the effect of drugs in anaesthesia, by the use of a new minimally parameterized Multiple-Input-Single-Output (MISO) Wiener model. As expected, this approach leads to a model whose parameters have a reduced physiological meaning. However, even for the clinical point of view, this is not a crucial issue since improved results are achieved in what concerns parameter adaptation and output signal tracking. Furthermore, continuous time parameters are estimated, a fact that facilitates physiological interpretation.

The input signals consist of the dosage of propofol and remifentanil. The human effect output signal used in the paper is the clinically-validated electroencephalogram-derived Bispectral Index Scale (BIS) [9]. It can be noted that the BIS is at the focal point of anaesthesia research today. Ranging from $100 \%$ (completely "awake" clinical state) to $0 \%$ (isoelectric EEG) the BIS is widely used as an indicator of the DoA. In a general surgery environment, the BIS should be kept between $40 \%$ and $60 \%$ to enable a faster emergence from anaesthesia [10] and a lower administered drug dosage [11]. The combined administration of hypnotics and analgesics for induction and maintenance of DoA is a common practice. The hypnotic is targeted to the loss of consciousness and prevents awareness, while the analgesic is given to supress pain. Indeed, the clinical advantage of this combined procedure is the synergistic increase in the expected effects of both drugs based on the nonlinear concentration-effect relationships of the individual agents [12]. This evidence is modeled by a nonlinear interaction function between the two drugs.

The present paper is structured as follows. Section II describes the state of the art of DoA modeling, while Section III presents both the linear and nonlinear parts of the new minimally parameterized model for the BIS response. In Section IV a batch identification algorithm is derived as an intermediate step, and the results of its application on the clinical database appears in Section V. In Section VI the Extended Kalman Filter algorithm is derived, followed by the presentation of results when applied to patient data in Section VII. Conclusions follow in Section VIII.

\section{DOA: STATE OF THE ART MODELS}

The most commonly used models in the literature to describe the PK and PD of the propofol (hypnotic) and remifentanil (analgesic) effects in the human body are described in this section. It should be stressed that these models will not be the ones used for the development of the identification methodologies of this study. These models are discussed here to point out the need for the development of new minimally parameterized models to match the parsimony principle of system identification [2].

\section{A. Linear part}

The PK models describe mixing dynamics of the drugs in the different theoretical compartments of the human body [13]. Both for propofol and remifentanil, a 3-compartment mammilary model is normally used [14], [15]. As illustrated in Fig. 1, the drug (considered as the system input) is intravenously administrated, entering in the main compartment with infusion rate $r(t)\left[\mathrm{mg} \mathrm{ml}^{-1} \mathrm{~min}^{-1}\right]$. The clearance of the drug, modeled by an outer flow of the main compartment $k_{10}\left[\mathrm{~min}^{-1}\right]$, and the transfer coefficients $k_{i j}\left[\mathrm{~min}^{-1}\right]$ from compartment $i$ to $j$ are patient dependent parameters.

By direct deduction from mass balances between compartments, and assuming that for each compartment $i$, a concentration $x_{i}(t)\left[\mathrm{mg} \mathrm{ml}^{-1}\right]$ of drug is present, the state space representation that is commonly used becomes 


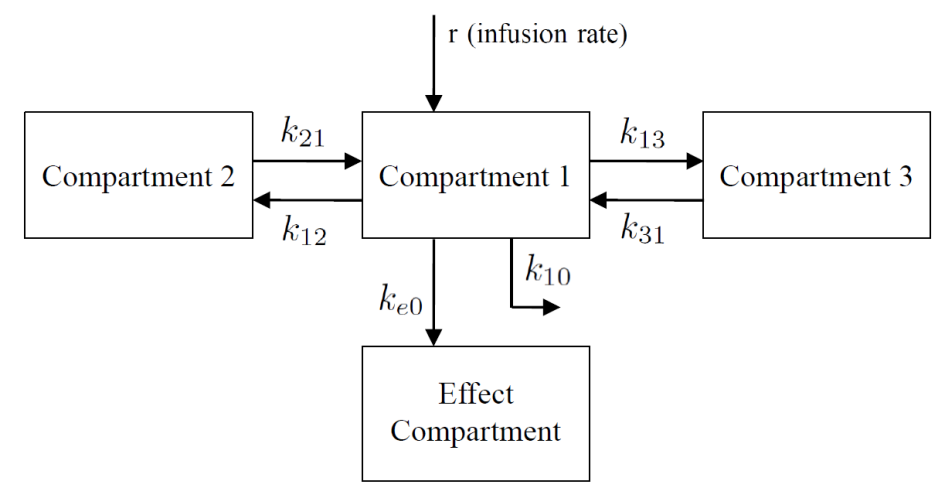

Fig. 1. Mamillary model for both propofol and remifentanil PK

$$
\dot{x}(t)=\left(\begin{array}{c}
\dot{x}_{1}(t) \\
\dot{x}_{2}(t) \\
\dot{x}_{3}(t)
\end{array}\right)=\left(\begin{array}{ccc}
-\left(k_{10}+k_{12}+k_{13}\right) & k_{21} \frac{V_{2}}{V_{1}} & k_{31} \frac{V_{3}}{V_{1}} \\
k_{12} \frac{V_{1}}{V_{2}} & -k_{21} & 0 \\
k_{13} \frac{V_{1}}{V_{3}} & 0 & -k_{31}
\end{array}\right) x(t)+\left(\begin{array}{l}
1 \\
0 \\
0
\end{array}\right) r(t),
$$

where $V_{i}[\mathrm{ml}]$ represents the volume of compartment $i$.

The PD nonlinearity relates the drug concentration with the effect, DoA, and has a first linear dynamic part approximated by a first order differential equation [16]

$$
\dot{x}_{e}(t)=k_{e 0}\left(x_{1}(t)-x_{e}(t)\right),
$$

where $k_{e 0}$ is the inverse of the equilibrium time between the central compartment (compartment 1 in Fig. 1) and the effect compartment, and $x_{e}(t)\left[\mathrm{mg} \mathrm{ml}^{-1}\right]$ is the effect compartment concentration (which cannot be measured).

In clinical practice, population models are applied to infer the response of each patient to a certain drug administration profile. For propofol, the parameters from Marsh [17] or from Schnider [18] are usually used, whereas for remifentanil, the parameters from Minto [15] are commonly applied.

\section{B. Nonlinear interaction}

This section describes the widely accepted nonlinear model for the interaction between propofol and remifentanil [19]. It relates the effect concentration of both drugs with the electroencephalographic parameter values e.g. BIS. Both effect concentrations $x_{e}^{\text {prop }}(t)$ and $x_{e}^{r e m i}(t)$ are first normalized with respect to their concentration at half the maximal effect ( $E C_{50}^{\text {prop }}$ and $E C_{50}^{\text {remi }}$, respectively), i.e.

$$
U_{\text {prop }}(t)=\frac{x_{e}^{\text {prop }}(t)}{E C_{50}^{\text {prop }}}, \quad U_{\text {remi }}(t)=\frac{x_{e}^{\text {remi }}(t)}{E C_{50}^{\text {remi }}} .
$$

As mentioned before, since the interaction of propofol and remifentanil is not purely additive, the potency of the drug mixture is modeled as follows

$$
\phi=\frac{U_{\text {prop }}(t)}{U_{\text {prop }}(t)+U_{\text {remi }}(t)},
$$

where, by definition, $\phi$ ranges from 0 (remifentanil only) and 1 (propofol only). The nonlinear concentrationresponse relationship for any ratio of the two drugs can then be described by a generalized Hill equation as 


$$
y(t)=y_{0}\left(1-\frac{\left(\frac{U_{p r o p}(t)+U_{r e m i}(t)}{U_{50}(\phi)}\right)^{\gamma}}{1+\left(\frac{U_{p r o p}(t)+U_{r e m i}(t)}{U_{50}(\phi)}\right)^{\gamma}}\right),
$$

where $y_{0}$ is the effect at zero concentration, $\gamma$ controls the steepness of the nonlinear concentrationresponse relation, and $U_{50}(\phi)$ is the number of units associated with $50 \%$ of the maximum effect of both drugs at ratio $\phi$. In [19] a quadratic polynomial was proposed for the expression of $U_{50}(\phi)$ :

$$
U_{50}(\phi)=1-\beta \phi+\beta \phi^{2} .
$$

In common clinical practice, the parameters $\gamma, \beta, E C_{50}^{\text {prop }}$ and $E C_{50}^{r e m i}$ and the linear parameters $k_{i j}$ and $k_{e 0}$ of (1) and (2) are usually guessed based on population model distributions and on the clinicians' expertise. The result is a model depending on many continuous time parameters.

\section{MINIMALLY PARAMETERIZED MODEL}

A new MISO Wiener model with a minimal number of parameters describing the joint effect of propofol and remifentanil in the human body is presented in this section. Models are first built using a continuous-time description and then sampled with a zero-order hold strategy. This makes it possible to derive algorithms that estimate the underlying continuous time parameters, exploiting the intrinsic feature of having a minimal number of parameters.

\section{A. Linear block}

By inspection of the model structure, the plasmatic concentration (in compartment 1 of Fig. 1) and the effect concentration coming from population studies for both propofol [18] and remifentanil [15], a third-order continuous time model is proposed for the linear dynamics of each drug. It can be noted that this approach was also very successful in [8].

Propofol linear dynamics is hence modeled by

$$
\hat{X}_{e}^{p r o p}(s, \alpha)=\frac{k_{1} k_{2} k_{3} \alpha^{3}}{\left(s+k_{1} \alpha\right)\left(s+k_{2} \alpha\right)\left(s+k_{3} \alpha\right)} R^{\text {prop }}(s),
$$

where $\hat{X}_{e}^{\text {prop }}(s, \alpha)$ is the Laplace transform of the output from the model linear dynamic part for propofol $\hat{x}_{e}^{\text {prop }}(t, \alpha)$ and $R^{\text {prop }}(s)$ is the Laplace transform of the input signal $r^{\text {prop }}(t)$. The pole location $\alpha$ is selected as the parameter to be identified.

Remifentanil linear dynamics is similarly modeled by

$$
\hat{X}_{e}^{r e m i}(s, \eta)=\frac{l_{1} l_{2} l_{3} \eta^{3}}{\left(s+l_{1} \eta\right)\left(s+l_{2} \eta\right)\left(s+l_{3} \eta\right)} R^{r e m i}(s)
$$

where $\hat{X}_{e}^{r e m i}(s, \eta)$ is the Laplace transform of the output from the model for remifentanil $\hat{x}_{e}^{r e m i}(t, \eta)$ and $R^{r e m i}(s)$ is the Laplace transform of the input signal $r^{r e m i}(t)$. The pole location $\eta$ is selected as the parameter to be identified. 


\section{B. Nonlinear block}

Using the model in (5) as starting point, a new formulation for the nonlinearity is now proposed. According to (6),

$$
\frac{\hat{U}_{p r o p}(t, \alpha)+\hat{U}_{r e m i}(t, \eta)}{U_{50}(\phi)}=\frac{\hat{U}_{p r o p}(t, \alpha)+\hat{U}_{r e m i}(t, \eta)}{1-\beta \phi+\beta \phi^{2}} .
$$

Hence, to motivate the new model, consider $|r|=\left|\beta \phi-\beta \phi^{2}\right|$ to be less than 1 and exploit the Taylor series expansion

$$
\frac{1}{1-r}=1+r+r^{2}+r^{3}+\ldots
$$

Then, neglecting the high-order terms, and substituting $\phi$ according to (4), it follows that

$$
\begin{aligned}
& \frac{\hat{U}_{\text {prop }}(t, \alpha)+\hat{U}_{\text {remi }}(t, \eta)}{U_{50}(\phi)} \\
\approx & \left(\hat{U}_{\text {prop }}(t, \alpha)+\hat{U}_{\text {remi }}(t, \eta)\right)\left(1+\beta \frac{\hat{U}_{\text {prop }}(t, \alpha)}{\hat{U}_{\text {prop }}(t, \alpha)+\hat{U}_{\text {remi }}(t, \eta)}\right) \\
= & \hat{U}_{\text {remi }}(t, \eta)+(1+\beta) \hat{U}_{\text {prop }}(t, \alpha) \\
= & \hat{U}_{\text {remi }}(t, \eta)+m \hat{U}_{\text {prop }}(t, \alpha) .
\end{aligned}
$$

Here $m=1+\beta$ is selected as one of the parameters to be identified. Furthermore, following (3),

$$
\hat{U}_{\text {prop }}(t, \alpha)=\frac{\hat{x}_{e}^{\text {prop }}(t, \alpha)}{E C_{50}^{\text {prop }}}, \quad \hat{U}_{r e m i}(t, \eta)=\frac{\hat{x}_{e}^{r e m i}(t, \eta)}{E C_{50}^{r e m i}} .
$$

Hence, (5) is substituted by the new formulation:

$$
\hat{y}(t)=\frac{y_{0}}{1+\left(\hat{U}_{\text {remi }}(t, \eta)+m \hat{U}_{\text {prop }}(t, \alpha)\right)^{\gamma}} .
$$

\section{Sampling}

In order to implement the proposed model structure in discrete-time identification algorithms, the continuous-time representations of (7) and (8) have to be sampled. For that purpose the representations (7) and (8) are transformed into a joint continuous time state-space form (15) describing the linear dynamics of each drug.

Due to the fact that the synergistic interaction between propofol and remifentanil of (12) is no more nonlinear, the linear state-space output $\hat{z}(t)$ will be the result from the linear weighted sum in (12)

$$
\hat{z}(t)=\hat{U}_{\text {remi }}(t, \eta)+m \hat{U}_{\text {prop }}(t, \alpha) .
$$

Hence, the continuous-time representation becomes

$$
\begin{array}{ccc}
\dot{\hat{x}}(t) & = & A(\alpha, \eta) \hat{x}(t)+B r(t), \\
\hat{z}(t) & = & C(\theta) \hat{x}(t)
\end{array}
$$

with

$$
A(\alpha, \eta)=\left(\begin{array}{ll}
A_{p}(\alpha) & \underline{0}_{(3 \times 3)} \\
\underline{0}_{(3 \times 3)} & A_{r}(\eta)
\end{array}\right)
$$

where $A_{p}(\alpha) \in \mathbb{R}^{3 \times 3}$ is the system matrix for propofol (deduced from (7)) and $A_{r}(\eta) \in \mathbb{R}^{3 \times 3}$ is the system matrix for remifentanil (deduced from (8)). In (15), $B, r(t)$ and $C(\theta)$ are as following

$$
B=\left(\begin{array}{llllll}
1 & 0 & 0 & 0 & 0 & 0 \\
0 & 0 & 0 & 1 & 0 & 0
\end{array}\right)^{T}
$$




$$
\begin{gathered}
r(t)=\left(\begin{array}{lllll}
r^{\text {prop }}(t) & r^{r e m i}(t)
\end{array}\right)^{T}, \\
C(\theta)=\left(\begin{array}{llllll}
0 & 0 & m \frac{k_{1} k_{2} k_{3} \alpha^{3}}{E C_{50}^{p r o p}} & 0 & 0 & \frac{l_{1} l_{2} l_{3} \eta^{3}}{E C_{50}^{r e m i}}
\end{array}\right) .
\end{gathered}
$$

Finally, the nonlinear output is obtained from (13) as

$$
\hat{y}(t)=\frac{y_{0}}{1+(\hat{z}(t))^{\gamma}} .
$$

The zero-order hold method [20] was used with sampling instant $t_{k}=k h$. For BIS, $h$ is usually equal to 5 seconds. This value is dependent on the sampling rate of the measurement devices. The discrete time model becomes

$$
\begin{aligned}
\hat{x}(k h+h) & =\Phi(\alpha, \eta) \hat{x}(k h)+\Gamma(\alpha, \eta) r(k h) \\
\hat{z}(k h) & =C(\theta) \hat{x}(k h) \\
\hat{y}(k h)= & \frac{y_{0}}{1+(\hat{z}(k h))^{\gamma}} \\
\Phi(\alpha, \eta) & =e^{A(\alpha, \eta) h} \\
\Gamma(\alpha, \eta) & =\int_{0}^{h} e^{A(\alpha, \eta) s} d s B .
\end{aligned}
$$

Here, $r(k h) \in \mathbb{R}^{2 \times 1}$ is the input (piecewise constant drug dose infusion for both propofol and remifentanil, according to (18)), $\hat{x}(k h) \in \mathbb{R}^{6 \times 1}$ the discrete model state-vector, $\hat{z}(k h) \in \mathbb{R}$ is the linear output (joint effect resulting from the interaction between the two drugs), $\Phi(\alpha, \eta) \in \mathbb{R}^{6 \times 6}$ and $\Gamma \in \mathbb{R}^{6 \times 1}$, being the sampled system matrices. The model output is given by $\hat{y}(k h)$.

For the purpose of implementing the prediction error method, the polynomial counterpart to (21) is more suitable to use. According to [20], the pulse transfer operator corresponding to (21) is given by

$$
H\left(q^{-1}, \theta\right)=C(\theta)(q I-\Phi(\alpha, \eta))^{-1} \Gamma(\alpha, \eta)=\frac{B\left(q^{-1}, \theta\right)}{A\left(q^{-1} \theta\right)} .
$$

where $q$ is the shift operator $(q u(k h)=u(k h+h))$.

The model output from the linear part can then be represented as

$$
\hat{z}(t)=\frac{B\left(q^{-1}, \theta\right)}{A\left(q^{-1}, \theta\right)} u(t) .
$$

The sampling does not affect the nonlinear block, hence (20) can be used as it is.

\section{Total model}

For the new proposed model several quantities are fixed or determined offline. Similarly as in [8], the parameters $k_{i}$ and $l_{i}, i=1, \ldots, 3$ are determined by the use of batch identification over a large database of patient data (see Section IV). Note that $k_{1}=l_{1}=1$ needs to hold to get a unique parameterization. The normalizing constants $E C_{50}^{\text {prop }}$ and $E C_{50}^{\text {remi }}$ are also fixed (similarly to the work in [21]). When using BIS as the parameter to infer the DoA, $y_{0}$ in the nonlinear block is equal to 97.7 due to monitor restrictions.

The linear part of the system is then parameterized in the frequency domain as (7) and (8) with the systems inputs being the propofol rate $r_{\text {prop }}(t)$ and remifentanil rate $r_{\text {remi }}(t)$, respectively. After normalizing the outputs of the linear part $\hat{x}_{e}^{\text {prop }}(t, \alpha)$ and $\hat{x}_{e}^{r e m i}(t, \eta)$ with respect to $E C_{50}^{\text {prop }}$ and $E C_{50}^{r e m i}$ (according to (12)), the model output $\hat{y}(t)$ is obtained through the nonlinear function (13). The total parameter vector to be identified is then

$$
\theta=\left(\begin{array}{llll}
\alpha & \eta & m & \gamma
\end{array}\right)^{T}
$$




\section{BATCH IDENTIFICATION ALGORITHM: PREDICTION ERROR METHOD}

The prediction error method determines $\theta$ so that the prediction error

$$
\varepsilon(t, \theta)=y(t)-\hat{y}(t \mid \theta) .
$$

becomes as small as possible. Note that $y(t)$ is the measured output value and $\hat{y}(t \mid \theta)$ is the predicted output, based on parameter vector $\theta$.

\section{A. Gradient}

The gradient of the prediction error (26) is of central importance to the prediction error identification method. It is given by:

$$
\begin{aligned}
& \psi(t, \theta)=-\left(\frac{\partial \varepsilon(t, \theta)}{\partial \theta}\right)^{T} \\
& =\left(\frac{\partial \hat{y}(t \mid \theta)}{\partial \theta}\right)^{T} \\
& =\left(\begin{array}{llll}
\frac{\partial \hat{y}(t \mid \theta)}{\partial \alpha} & \frac{\partial \hat{y}(t \mid \theta)}{\partial \eta} & \frac{\partial \hat{y}(t \mid \theta)}{\partial m} & \frac{\partial \hat{y}(t \mid \theta)}{\partial \gamma}
\end{array}\right)^{T} \\
& =\left(\frac{\partial \hat{y}(t \mid \theta)}{\partial \hat{U}_{\text {prop }}(t, \alpha)} \frac{\partial \hat{U}_{\text {prop }}(t, \alpha)}{\partial \alpha} \quad \frac{\partial \hat{y}(t \mid \theta)}{\partial \hat{U}_{\text {remi }}(t, \eta)} \frac{\partial \hat{U}_{r e m i}(t, \eta)}{\partial \eta} \quad \frac{\partial \hat{y}(t \mid \theta)}{\partial m} \quad \frac{\partial \hat{y}(t \mid \theta)}{\partial \gamma}\right)^{T}
\end{aligned}
$$

\section{B. Criterion and search direction}

In order to derive the prediction error method, the following criterion is introduced [2]:

$$
V(\theta)=\frac{1}{N} \sum_{t=1}^{N} \varepsilon^{2}(t, \theta),
$$

where $N$ is the total number of data points and $\varepsilon(t, \theta)$ is the prediction error.

The minimization of (28) is then performed using the numerical Gauss-Newton method [2]:

$$
\hat{\theta}^{(k+1)}=\hat{\theta}^{(k)}-\beta_{k}\left[V^{\prime \prime}\left(\hat{\theta}^{(k)}\right)\right]^{-1} V^{\prime}\left(\hat{\theta}^{(k)}\right)^{T},
$$

where $\hat{\theta}^{(k)}$ denotes the $k$ th iteration in the search. The sequence of scalars $\beta_{k}$ is used to control the step lenght. The derivatives of $V(\theta)$ can be found as:

$$
\begin{aligned}
V^{\prime}(\theta)= & -\frac{2}{N} \sum_{t=1}^{N} \varepsilon^{T}(t, \theta) \psi^{T}(t, \theta), \\
V^{\prime \prime}(\theta)= & \frac{2}{N} \sum_{t=1}^{N} \psi(t, \theta) \psi^{T}(t, \theta) \\
& +\frac{2}{N} \sum_{t=1}^{N} \varepsilon(t, \theta) \frac{\partial}{\partial \theta} \psi^{T}(t, \theta) \\
\approx & \frac{2}{N} \sum_{t=1}^{N} \psi(t, \theta) \psi^{T}(t, \theta)
\end{aligned}
$$


The approximation in (31) is justified in [2] and is supported by the fact that, at the global minimum point $\varepsilon(t, \theta)$ becomes assymptotically white noise which is independent of $\psi(t, \theta)$. As a consequence, the Gauss-Newton method has a linear convergence instead of quadratic, which would be the case if the approximation had not been performed (i.e. using the Newton-Raphson method).

\section{Projection algorithm}

Regarding the linear block of the Wiener model in study, the application of a projection algorithm is needed to keep the model assymptotically stable. For that purpose, the poles of the transfer function (24) are monitored by the use of the following projection algorithm for the parameter $\alpha$ and $\eta$ :

$$
\begin{aligned}
& \alpha^{(k+1)}=\left\{\begin{array}{ccc}
\alpha^{(k+1)} & \text { if } & \alpha^{(k+1)}>\delta>0 \\
\alpha^{(k)} & \text { if } & \alpha^{(k+1)} \leq \delta
\end{array}\right. \\
& \eta^{(k+1)}=\left\{\begin{array}{clc}
\eta^{(k+1)} & \text { if } & \eta^{(k+1)}>\delta>0 \\
\eta^{(k)} & \text { if } & \eta^{(k+1)} \leq \delta
\end{array}\right.
\end{aligned}
$$

According to [19], the interaction between propofol and remifentanil is supraadditive. It is then natural to assume that $m$ in (12) is lower bounded by zero. As consequence, the following projection algorithm was used for the parameter $m$ :

$$
m^{(k+1)}=\left\{\begin{array}{ccc}
m^{(k+1)} & \text { if } & m^{(k+1)}>\delta>0 \\
m^{(k)} & \text { if } & m^{(k+1)} \leq \delta
\end{array}\right.
$$

For the nonlinear block (13), it is necessary to assure that $\gamma$ does not reach negative values. The following projection algorithm was used for the parameter $\gamma$ :

$$
\gamma^{(k+1)}=\left\{\begin{array}{ccc}
\gamma^{(k+1)} & \text { if } & \gamma^{(k+1)}>\delta>0 \\
\gamma^{(k)} & \text { if } & \gamma^{(k+1)} \leq \delta
\end{array}\right.
$$

Both updatings are hence stopped if the new parameter updates are outside the allowable range.

\section{Prediction error algorithm}

The Prediction error algorithm can now be summarized using the formulas defined above. Note that a numerical differentiation is used. The reason is that the mathematical expressions for the derivatives of (24) with respect to $\alpha$ and $\eta$ become very complicated.

$$
\begin{aligned}
& \text { for } k=0 \text { to } K \\
& \hat{\theta}=\hat{\theta}^{(k)} \\
& \hat{z}(t)=\frac{B\left(q^{-1}, \hat{\theta}\right)}{A\left(q^{-1}, \hat{\theta}\right)} u(t) \\
& \hat{y}(t \mid \hat{\theta})=\frac{y_{0}}{1+\left(\hat{U}_{\text {remi }}(t, \hat{\eta})+\hat{m} \hat{U}_{\text {prop }}(t, \hat{\alpha})\right)^{\hat{\gamma}}} \\
& \varepsilon(t, \hat{\theta})=y(t)-\hat{y}(t \mid \hat{\theta})
\end{aligned}
$$




$$
\begin{aligned}
& \frac{\partial \hat{y}(t \mid \hat{\theta})}{\partial \hat{U}_{\text {prop }}(t, \hat{\alpha})}=\frac{-y_{0} \hat{m} \hat{\gamma}\left(\hat{U}_{\text {remi }}(t, \hat{\eta})+\hat{m} \hat{U}_{\text {prop }}(t, \hat{\alpha})\right)^{\hat{\gamma}-1}}{\left(1+\left(\hat{U}_{\text {remi }}(t, \hat{\eta})+\hat{m} \hat{U}_{\text {prop }}(t, \hat{\alpha})\right)^{\hat{\gamma}}\right)^{2}} \\
& \frac{\partial \hat{y}(t \mid \hat{\theta})}{\partial \hat{U}_{r e m i}(t, \hat{\eta})}=\frac{-y_{0} \hat{\gamma}\left(\hat{U}_{\text {remi }}(t, \hat{\eta})+\hat{m} \hat{U}_{\text {prop }}(t, \hat{\alpha})\right)^{\hat{\gamma}-1}}{\left(1+\left(\hat{U}_{\text {remi }}(t, \hat{\eta})+\hat{m} \hat{U}_{\text {prop }}(t, \hat{\alpha})\right)^{\hat{\gamma}}\right)^{2}} \\
& \frac{\partial \hat{y}(t \mid \hat{\theta})}{\partial \hat{m}}=\frac{-y_{0} \hat{U}_{\text {prop }}(t, \hat{\alpha}) \hat{\gamma}\left(\hat{U}_{\text {remi }}(t, \hat{\eta})+\hat{m} \hat{U}_{\text {prop }}(t, \hat{\alpha})\right)^{\hat{\gamma}-1}}{\left(1+\left(\hat{U}_{\text {remi }}(t, \hat{\eta})+\hat{m} \hat{U}_{\text {prop }}(t, \hat{\alpha})\right)^{\hat{\gamma}}\right)^{2}} \\
& \frac{\partial \hat{y}(t \mid \hat{\theta})}{\partial \hat{\gamma}}=\frac{-y_{0} \log \left(\hat{U}_{\text {remi }}(t, \hat{\eta})+\hat{m} \hat{U}_{\text {prop }}(t, \hat{\alpha})\right)\left(\hat{U}_{\text {remi }}(t, \hat{\eta})+\hat{m} \hat{U}_{\text {prop }}(t, \hat{\alpha})\right)^{\hat{\gamma}}}{\left(1+\left(\hat{U}_{\text {remi }}(t, \hat{\eta})+\hat{m} \hat{U}_{\text {prop }}(t, \hat{\alpha})\right)^{\hat{\gamma}}\right)^{2}} \\
& \frac{\partial \hat{U}_{\text {prop }}(t, \hat{\alpha})}{\partial \hat{\alpha}}=\frac{\hat{U}_{\text {prop }}(t, \hat{\alpha}+\Delta \hat{\alpha})-\hat{U}_{\text {prop }}(t, \hat{\alpha})}{\Delta \hat{\alpha}} \\
& \frac{\partial \hat{U}_{r e m i}(t, \hat{\eta})}{\partial \hat{\eta}}=\frac{\hat{U}_{r e m i}(t, \hat{\eta}+\Delta \hat{\eta})-\hat{U}_{r e m i}(t, \hat{\eta})}{\Delta \hat{\eta}} \\
& \psi(t, \hat{\theta})=-\left(\frac{\partial \varepsilon(t, \hat{\theta})}{\partial \hat{\theta}}\right)^{T} \\
& =\left(\frac{\partial \hat{y}(t \mid \hat{\theta})}{\partial \hat{\theta}}\right)^{T} \\
& =\left(\begin{array}{llll}
\frac{\partial \hat{y}(t \mid \hat{\theta})}{\partial \hat{\alpha}} & \frac{\partial \hat{y}(t \mid \hat{\theta})}{\partial \hat{\eta}} & \frac{\partial \hat{y}(t \mid \hat{\theta})}{\partial \hat{m}} & \frac{\partial \hat{y}(t \mid \hat{\theta})}{\partial \hat{\gamma}}
\end{array}\right)^{T} \\
& =\left(\frac{\partial \hat{y}(t \mid \hat{\theta})}{\partial \hat{U}_{\text {prop }}(t, \hat{\alpha})} \frac{\partial \hat{U}_{\text {prop }}(t, \hat{\alpha})}{\partial \hat{\alpha}} \quad \frac{\partial \hat{y}(t \mid \hat{\theta})}{\partial \hat{U}_{\text {remi }}(t, \hat{\eta})} \frac{\partial \hat{U}_{\text {remi }}(t, \hat{\eta})}{\partial \hat{\eta}} \quad \frac{\partial \hat{y}(t \mid \hat{\theta})}{\partial \hat{m}} \quad \frac{\partial \hat{y}(t \mid \hat{\theta})}{\partial \hat{\gamma}}\right)^{T} \\
& V^{\prime}(\hat{\theta})=-\frac{2}{N} \sum_{t=1}^{N} \varepsilon^{T}(t, \hat{\theta}) \psi^{T}(t, \hat{\theta}) \\
& V^{\prime \prime}(\hat{\theta}) \approx \frac{2}{N} \sum_{t=1}^{N} \psi(t, \hat{\theta}) \psi^{T}(t, \hat{\theta})
\end{aligned}
$$




$$
\hat{\theta}^{(k+1)}=\hat{\theta}^{(k)}-\beta_{k}\left[V^{\prime \prime}\left(\hat{\theta}^{(k)}\right)\right]^{-1} V^{\prime}\left(\hat{\theta}^{(k)}\right)^{T}
$$

end

The steps $\Delta \hat{\alpha}$ and $\Delta \hat{\eta}$ used for the differentiation are selected to be small.

\section{OPTIMIZATION OF THE POLE LOCATION OF THE NEW MODEL}

In order to choose a combination of $k_{i}^{\prime} s$ and $l_{i}^{\prime} s$ (Section III-A) that leads to the best identification results, the PEM described in Section IV-D was applied to the signals from the seven real collected cases present in the available database. The signal time window for evaluation (of lenght $M$ ) started at the beginning of the induction phase and stopped at the end of the infusion $\left(t=t^{*}\right)$. For the same patient, a combination of $\left(k_{2}, k_{3}, l_{2}, l_{3}\right)$ ranging from $k_{i}=2$ to $k_{i}=10$ and $l_{i}=2$ to $l_{i}=10,\{i=2,3\}$ was tested. The same procedure was applied to every patient. The number of total algorithm iterations in each trial was 200, to ensure convergence of the identified parameters. The normalized sum of the squared prediction error $\varepsilon(t, \hat{\theta})$ for each combination $\left(k_{2}, k_{3}, l_{2}, l_{3}\right)$ and for each identified patient $j$ was calculated:

$$
\varepsilon_{j}\left(k_{2}, k_{3}, l_{2}, l_{3}\right)=\frac{1}{M} \sum_{t=0 \min }^{t^{*}}(\varepsilon(t, \hat{\theta}))^{2} .
$$

To perform a statistical analysis of the results coming from simulations with different $\left(k_{2}, k_{3}, l_{2}, l_{3}\right)$ combinations, a normalized sum among the total number of patients was calculated to obtain a single performance error value $\Psi\left(k_{2}, k_{3}, l_{2}, l_{3}\right)$ for each $\left(k_{2}, k_{3}, l_{2}, l_{3}\right)$ combination:

$$
\Psi\left(k_{2}, k_{3}, l_{2}, l_{3}\right)=\frac{1}{7} \sum_{j=1}^{7} \varepsilon_{j}\left(k_{2}, k_{3}, l_{2}, l_{3}\right)
$$

The minimum of the cost function $\Psi\left(k_{2}, k_{3}, l_{2}, l_{3}\right)$ corresponds to the choice of $k_{2}=9, k_{3}=10$, $l_{2}=2$ and $l_{3}=3$.

In order to graphically illustrate this minimum, $k_{2}$ and $k_{3}$ were fixed in 9 and 10 , respectively and the values of $\Psi\left(9,10, l_{2}, l_{3}\right)$ were plotted for the different combinations of $l_{2}$ and $l_{3}$, as shown in Fig. 2 .

By analyzing the surface in Fig. 2 it was found that the minimum normalized error for each $\left(9,10, l_{2}, l_{3}\right)$ combination in the patient database appeared for $l_{2}=2$ and $l_{3}=3$ or vice-versa. From here on, all the tests performed on the database will use the following values: $k_{2}=9, k_{3}=10, l_{2}=2$, and $l_{3}=3$.

\section{RECURSIVE IDENTIFICATION ALGORITHM: EXTENDED KALMAN FILTER}

The main reason for the use of the EKF is the possibility to obtain the parameter estimates along with system state estimates online. In comparison with other recursive identification techniques, it enables the independent tuning using the covariance matrix of both the process and measurement noise, thereby giving rise to an independent tuning of the convergence speed for each parameter. To describe the EKF the general discrete time nonlinear model

$$
\begin{aligned}
\hat{x}(k+1) & =f(k, \hat{x}(k), r(k))+g(k, \hat{x}(k)) v(k) \\
\hat{y}(k) & =h(k, \hat{x}(k))+e(k),
\end{aligned}
$$

is used, where $v(k)$ and $e(k)$ are mutually independent Gaussian white noise sequences with zero mean and covariances given by $R_{1}(k)$ and $R_{2}(k)$, respectively. The EKF algorithm can then be summarized as follows [22]: 


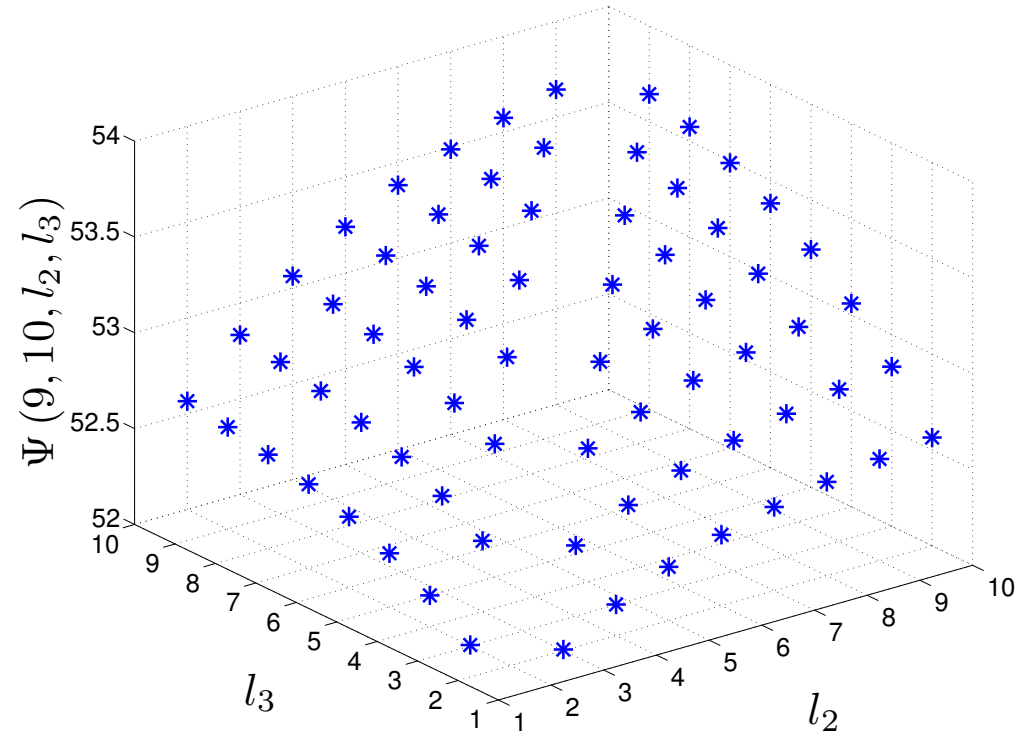

Fig. 2. Normalized performance error values for all combinations of $\left(9,10, l_{2}, l_{3}\right)$ from 2 to 10 . The values come from simulations performed in the database of 7 real patients with the PEM described in Section IV.

$$
\begin{aligned}
H(k)= & \left.\frac{\partial h(k, x)}{\partial x}\right|_{x=\hat{x}(k \mid k-1)} \\
K(k)= & P(k \mid k-1) H^{T}(k) \\
& \times\left[H(k) P(k \mid k-1) H^{T}(k)+R_{2}(k)\right]^{-1} \\
\hat{x}(k \mid k)= & \hat{x}(k \mid k-1)+K(k)[y(k)-h(k, \hat{x}(k \mid k-1))] \\
P(k \mid k)= & P(k \mid k-1)-K(k) H(k) P(k \mid k-1) \\
\hat{x}(k+1 \mid k)= & f(k, \hat{x}(k \mid k), r(k)) \\
F(k)= & \left.\frac{\partial f(k, x)}{\partial x}\right|_{x=\hat{x}(k \mid k)} \\
G(k)= & \left.g(k, x)\right|_{x=\hat{x}(k \mid k)} \\
P(k+1 \mid k)= & F(k) P(k \mid k) F^{T}(k)+G(k) R_{1}(k) G^{T}(k)
\end{aligned}
$$

\section{A. Discrete time model structural aspects}

A common strategy to enable the estimation of model parameters with the EKF is the introduction of an augmented state [2]. Hence, the model for the EKF estimation brings together the sampled model (21) and a random walk model for the parameter estimates [2]. Using (29), the augmented state vector 
becomes

$$
\hat{\bar{x}}(k h+h)=\left(\begin{array}{c}
\hat{\bar{x}}_{1}(k h+h) \\
\vdots \\
\hat{\bar{x}}_{6}(k h+h) \\
\hat{\bar{x}}_{7}(k h+h) \\
\vdots \\
\hat{\bar{x}}_{10}(k h+h)
\end{array}\right)=\left(\begin{array}{c}
\hat{x}_{1}(k h+h) \\
\vdots \\
\hat{x}_{6}(k h+h) \\
\hat{\alpha}(k h+h) \\
\vdots \\
\hat{\gamma}(k h+h)
\end{array}\right) .
$$

Hence, the augmented state-space model becomes

$$
\begin{aligned}
\hat{\bar{x}}(k h+h)= & \left(\begin{array}{cc}
\Phi(\alpha, \eta) & \underline{0}(6 \times 4) \\
\underline{0}_{(4 \times 6)} & I
\end{array}\right)\left(\begin{array}{c}
\hat{x}(k h) \\
\hat{\theta}(k h)
\end{array}\right) \\
& +\left(\begin{array}{c}
\Gamma(\alpha, \eta) \\
\underline{0}_{(4 \times 1)}
\end{array}\right) r(k h)+w(k h) \\
\equiv & \left(\begin{array}{c}
f_{1}(k h, \hat{\bar{x}}(k h), r(k h)) \\
f_{2}(k h, \hat{\bar{x}}(k h), r(k h)) \\
\vdots \\
f_{10}(k h, \hat{\bar{x}}(k h), r(k h))
\end{array}\right)+w(k h) \\
\equiv & f(k h, \hat{\bar{x}}(k h), r(k h))+w(k h) . \\
\hat{y}(k h)= & \frac{1+(\bar{C}(\theta) \hat{\bar{x}}(k h))^{\hat{x}_{10}(k h)}}{1+\hat{\bar{x}}(k h))+e(k h),} \\
\equiv & h\left(k h, y_{0}\right) \\
\bar{C}(\theta)= & (C(\theta) \quad 0 \quad 0 \quad 0 \quad 0) .
\end{aligned}
$$

\section{B. Linearization and Initialization}

In the EKF algorithm structure (39), it is necessary to linearize both $f(k, x)$ and $h(k, x)$. Both linearizations were performed analytically. The formulas for $F(k h)$ and $H(k h)$ are not shown here due to their complexity.

After having formulated all the quantities needed to set up the EKF, the initial values for the variables must be specified. The initial values for the states were

$$
\begin{aligned}
& \hat{\bar{x}}(0 \mid-1)=\left(\begin{array}{lllll}
0.0 & 0.0 & 0.0 & 0.0 & 0.0
\end{array}\right. \\
& \left.\begin{array}{lllll}
0.0 & 0.2 & 0.2 & 0.6 & 1.0
\end{array}\right)^{T}
\end{aligned}
$$

Using simulations performed on the available database, it was found that the diagonal matrix $P(t \mid t-1)$ could be initialized as

$$
P(0 \mid-1)=\operatorname{diag}\left(\begin{array}{ccccc}
100^{2} & 10^{2} & 1^{2} & 100^{2} & 10^{2} \\
1 & 10 & 10 & 10 & 10
\end{array}\right)
$$




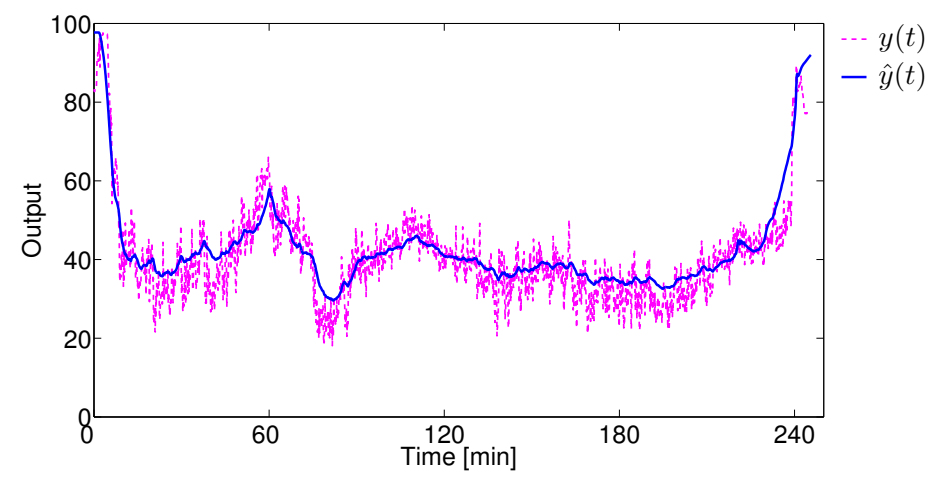

Fig. 3. The real measured BIS data (dashed line) plotted together with the simulated output model response (solid line), using the parameter estimates of the EKF.

Performing a similar empirical analysis on the simulated signals, the values for the diagonal covariance matrix $R_{1}$ and the covariance matrix $R_{2}$ were set to

$$
\begin{gathered}
R_{1}=\operatorname{diag}\left(\begin{array}{ccccc}
1000^{2} & 100^{2} & 10^{2} & 1000^{2} & 100^{2} \\
10^{2} & 10^{2} & 10^{2} & 10^{2} & 10^{2}
\end{array}\right) \\
R_{2}=10^{10}
\end{gathered}
$$

\section{RECURSIVE SIGNAL TRACKING RESULTS}

This section presents examples of the EKF performance applied to the new minimally parameterized model in Section III. Simulations were performed using the real BIS measurements from patients undergoing surgery. In these real cases in the database, the BIS signal was maintained around the level of 40. This was achieved by the action of the anesthetist who changed the infusion rates of both drugs according to the clinical goal.

The real measured BIS signal $y(t)$ and the EKF simulated BIS signal $\hat{y}(t)$ are illustrated in Fig. 3. The results show that the EKF simulated BIS signal tracks the real measured BIS signal excellently during the total time-window of this case study. More importantly, when the real BIS signal changes its behaviour, as at around minute 60 , the estimated model signal also tracks that behaviour. Sudden changes are also tracked by the simulated signal, e.g. the rapid drop in the signal the occurs right after this increase around minute 60. Furthermore, the algorithm is able to discard the noise effects present in the measured BIS, giving rise to a filtered signal able to catch the main trends of the real signal. This capability is obtained via the parameter estimates provided by the EKF algorithm. The time evolution of the parameter estimates $(\hat{\alpha}, \hat{\gamma}, \hat{m}$ and $\hat{\gamma})$ for this example are shown in Fig. 4. The input signals, propofol and remifentanil rates, that were present in this real case are the ones illustrated in Fig. 5.

It can be concluded from the plots that the new minimally parameterized Wiener model (41), (42) accurately describes the nonlinear system dynamics. By the use of the EKF strategy, model parameters can be extracted from the real BIS measurements. The tracking performance is excellent combining accurate modeling with very good noise suppression.

\section{CONCLUSIONS AND FUTURE WORK}

In this work, a new minimally parameterized MISO model for the human response to anaesthesia is designed. Furthermore, an online nonlinear identification strategy of that response using real measurements is proposed. The system inputs are the propofol (hypnotic drug) and remifentanil (analgesic drug) infusion rates, and the output is the Depth of Anaesthesia (DoA) quantitative measurement Bispectal Index Scale (BIS). The new model satisfactory describes the input-output system behaviour, having its 


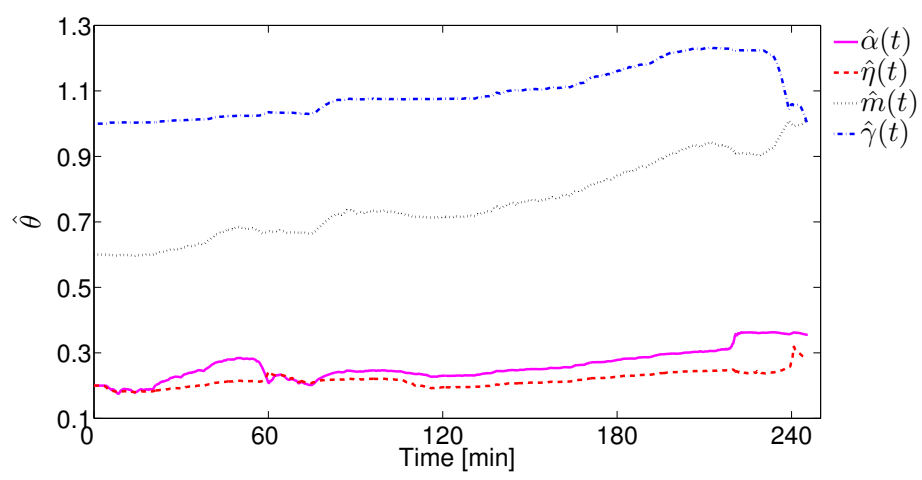

Fig. 4. The estimates of parameters $\alpha$ (solid line), $\eta$ (dashed line), $m$ (dotted line), and $\gamma$ (in dashe-dotted line) updated by the EKF for the identified real case in Fig. 3.

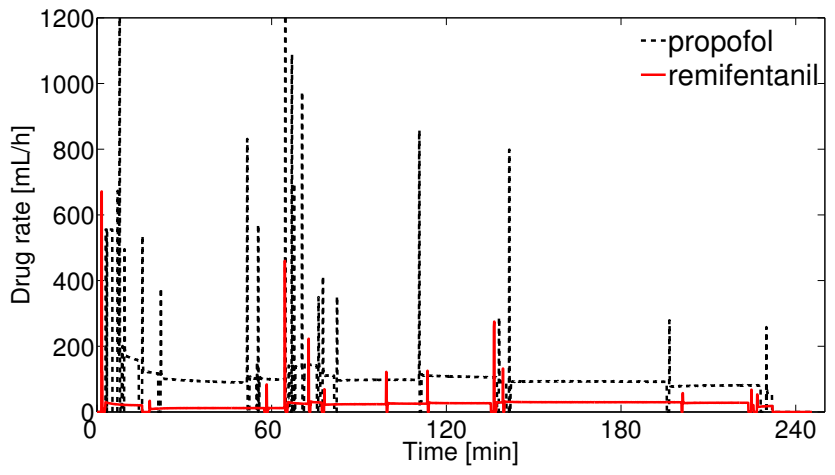

Fig. 5. The propofol rate (dashed line) and remifentanil rate (solid line) given to the patient with the measured BIS represented in Fig. 3.

parameters updated by the Extended Kalman Filter (EKF) identification algorithm that was derived from the new model.

The new model and algorithm address the poor excitation, the few output datapoints, and the high level of noise in the output signal. In addition to the very good BIS tracking and noise filtering achieved by the proposed identification algorithm, it can be noted that the parameter estimates are quite stationary. This is a very strong indication that the proposed minimal parameterization is in fact sufficient to describe the dynamics of the system, using a system identification perspective. This also indicates that difficulties can be expected when the standard models with many more parameters are used for identification purposes, a fact that has been reported in the literature. One other consequence of this is that the obtained parameter estimates can be expected to be very useful for controller design purposes, this being the ultimate goal for this research.

\section{ACKNOWLEDGMENTS}

The authors gratefully acknowledge the support of the Portuguese Science Foundation (FCT - Fundação para a Ciência e a Tecnologia), through the project IDeA - Integrated Design for Automation in Anaesthesia (reference PTDC/EEA- ACR/69288/2006). The stipend given by the Bernt Järmarks foundation for scientific research is also gratefully acknowledged.

\section{REFERENCES}

[1] J. M. Bailey and W. M. Haddad, "Drug dosing control in clinical pharmacology," Control Systems Magazine, IEEE, vol. 25, no. 2, pp. 35-51, April 2005. 
[2] T. Söderström and P. Stoica, System Identification. Hemel Hempstead, UK: Prentice-Hall, 1989.

[3] T. Wigren, "User choices and model validation in system identification using nonlinear Wiener models," in Proc. of the 13th IFAC Symposium on System Identification, Rotterdam, the Netherlands, 2003, pp. 863-868.

[4] - "Recursive prediction error identification method using the nonlinear Wiener model," Automatica, vol. 29, no. 4, pp. 1011-1025, 1993.

[5] H.-H. Lin, C. L. Beck, and M. J. Bloom, "On the use of multivariable piecewise-linear models for predicting human response to anesthesia," IEEE Trans. Biomed. Eng., pp. 1876-1887, Nov. 2004.

[6] H. Alonso, T. Mendonça, J. M. Lemos, and T. Wigren, "A simple model for the identification of drug effects," in Proc. IEEE International symposium on Intelligent Signal Processing (WISP'09), Budapest, Hungary, Aug. 2009.

[7] B. A. Costa, M. Silva, T. Mendonça, and J. M. Lemos, "Neuromuscular blockade nonlinear model identification," in Proc. Mediterranean Conference on Automation and Control (MED'09), Thessaloniki, Greece, June 2009, pp. 1137-1142.

[8] M. M. Silva, T. Mendonça, and T. Wigren, "Nonlinear identification of a minimal neuromuscular blockade model in anaesthesia," Dept. Information Technology, Uppsala University, Uppsala, Sweden, Tech. Rep. 2009-023, Available: http://www.it.uu.se/research/publications/reports/, Sep. 2009.

[9] J. C. Sigl and N. G. Chamoun, "An introduction to bispectral analysis for the electroencephalogram," J. Clin. Monit, vol. 10, pp. 392-404, 1994.

[10] D. Song, G. P. Joshi, and P. F. White, "Titration of volatile anesthetics using bispectral index facilitates recovery after ambulatory anesthesia," Anesthesiology, vol. 87(4), pp. 842-848, 1997.

[11] T. J. Gan, P. S. Glass, A. Windsor, F. Payne, C. Rosow, P. Sebel, and P. Manberg, "Bispectral index monitoring allows faster emergence and improved recovery from propofol, alfentanil, and nitrous oxide anesthesia," Anesthesiology, vol. 87(4), pp. 808-815, 1997.

[12] J. Vuyk, M. Mertens, E. Olofsen, A. Burm, and J. Bovill, "Propofol anesthesia and rational opioid selection: determination of optimal EC50-EC95 propofol-opioid concentrations that assure adequate anesthesia and a rapid return of consciousness," Anesthesiology, vol. 87(6), pp. 1549-1562, 1997.

[13] K. Godfrey, Compartmental models and their application. Academic Press, 1983.

[14] E. Gepts, F. Camu, I. Cockshott, and E. J. Douglas, "Disposition of propofol administered as constant rate intravenous infusions in humans," Anesthesia and Analgesia, vol. 66, pp. 1256-1263, 1987.

[15] C. F. Minto, T. W. Schnider, T. D. Egan, E. Youngs, H. J. Lemmens, P. L. Gambus, V. Billard, J. F. Hoke, K. H. Moore, D. J. Hermann, K. T. Muir, J. W. Mandema, and S. L. Shafer, "Influence of age and gender on the pharmacokinetics and pharmacodynamics of remifentanil. i. model development," Anesthesiology, vol. 86, pp. 10-23, 1997.

[16] S. L. Shafer, D. E. Lognecker, J. H. Tinker, and G. E. Morgan, Principles of pharmacokinetics and pharmacodynamics, 2nd ed., ser. Principles and Practice of Anesthesiology. New York: McGraw-Hill, 1998.

[17] B. Marsh, M. White, N. Norton, and G. N. Kenny, "Pharmacokinetic model driven infusion of propofol in children," Br. J. Anaesthesia, vol. 67, pp. 41-48, 1991.

[18] T. W. Schnider, C. F. Minto, P. L. Gambus, C. Andresen, D. B. Goodale, S. L. Shafer, and E. J. Youngs, "The influence of method of administration and covariates on the pharmacokinetics of propofol in adult volunteers," Anesthesiology, vol. 8, pp. 1170-1182, 1998.

[19] C. F. Minto, T. W. Schnider, T. G. Short, K. M. Gregg, A. Gentilini, and S. L. Shafer, "Response surface model for anesthetic drug interactions," Anesthesiology, vol. 92, pp. 1603-1616, 2000.

[20] K. J. Åström and B. Wittenmark, Computer-Controlled Systems. Englewood Cliffs, NJ: Prentice Hall, 1984.

[21] H. Alonso, T. Mendonça, and P. Rocha, "A hybrid method for parameter estimation and its application to biomedical systems," Computer Methods and Programs in Biomedicine, vol. 89, pp. 112-122, 2008.

[22] T. Söderström, Discrete-time Stochastic Systems. London, UK: Springer-Verlag, 2002. 